\title{
Turismo e Inovação em Espaços Rurais: estudo de caso da rede européia de turismo de aldeia
}

\author{
Tourism and Innovation in Rural Areas: the case study of the european \\ network of village tourism
}

\author{
Apolónia Rodrigues ${ }^{1}$
}

Áurea Rodrigues ${ }^{2}$

\begin{abstract}
Resumo
O comportamento do consumidor em turismo tem vindo a alterar-se intensamente levando ao desenvolvimento de um mercado mais especializado e sofisticado centrado nas actividades específicas que podem ser desenvolvidas a nível local. Neste contexto, os espaços rurais têm um apelo especial para os turistas por causa da mística ligada ao mundo rural e às características culturais, históricas, éticas e geográficas específicas destas áreas. As empresas turísticas em espaço rural são na sua maioria micro-empresas e PME's. O fenómeno da globalização criou uma grande pressão nas pequenas e médias empresas que apresentam dificuldade em formar unicidade num destino e em distribuir os seus produtos de uma forma abrangente. Assim, têm surgido nos últimos anos novas formas de interação entre os diferentes stakeholders, incluindo actores tanto do sector público como privado, assim como as populações locais, em que todos juntam esforços para o desenvolvimento e implementação de redes em destinos turísticos. Consequentemente, a construção de parcerias e a aplicação de acções e estratégias de marketing em conjunto podem reduzir o isolamento dos pequenos negócios permitindo o uso dos recursos de uma forma mais eficaz. No entanto, existe pouca literatura a evidenciar que a integração das PME's que operam no sector do turismo em redes possa ser usado como um factor de inovação que contribui para o desenvolvimento local. Neste artigo apresentamos um case study sobre os efeitos induzidos ao nível de inovação com a implementação de uma Rede Europeia de Turismo de Aldeia, em cinco regiões europeias.
\end{abstract}

Palavras-chave: inovação; redes; turismo rural; Europa

\begin{abstract}
The consumer behaviour in tourism has been changing leading to the development of a more specialized and sophisticated market focused on specific activities that can be developed at local level. In this context, rural areas have a special appeal to tourists because of the mystique attached to rural and cultural historical, ethical, characteristics of these specific geographic areas. The tourism businesses in rural areas are mostly micro-enterprises and SMEs. The phenomenon of globalization has created great pressure on small and medium enterprises, which have difficulty in forming a unified destination and distribute their products in a comprehensive manner. In this context, have emerged in recent years new forms
\end{abstract}

\footnotetext{
${ }^{1}$ Rede de Turismo de Aldeia do Alentejo, Genuineland. E-mail: genuineland@gmail.com

${ }^{2}$ DEGEI, Universidade de Aveiro Campus de Santiago, Portugal. Email: aurea@ua.pt
} 
of interaction between different stakeholders, including players from both the public and private sectors, as well as local populations, in which all join efforts for the development and implementation of networks in tourist destinations. In this context, the development of partnerships and implementation of joint strategic operations and marketing can reduce the isolation and allowing small businesses to use resources more efficiently. However, there is little literature to show that the integration of SMEs operating in the tourism sector in networks can be used as a source of innovation that contributes to local development. In this paper we present a case study on the impact of innovation with the implementation of a European Network of Village Tourism in five European regions.

Keywords: innovation; networks; rural tourism; Europe

\section{Introdução}

O comportamento do consumidor em turismo tem vindo a alterar-se intensamente levando ao desenvolvimento de um mercado mais especializado e sofisticado centrado nas actividades específicas que podem ser desenvolvidas a nível local (Novelli et al, 2006). Neste contexto, os espaços rurais têm um apelo especial para os turistas por causa da mística ligada ao mundo rural e às características culturais, históricas, éticas e geográficas específicas destas áreas (Sharpley, 2005; Fra, 2004; Cristóvão, 2002, Wilson et al, 2001, Dinis, 1999). As empresas turísticas em espaço rural são na sua maioria micro-empresas e PME’s.

Segundo Breda et al (2006) o fenómeno da globalização criou uma grande pressão nas pequenas e médias empresas, que apresentam dificuldade em formar unicidade num destino e em distribuir os seus produtos de uma forma abrangente. Assim, têm surgido nos últimos anos novas formas de iteração entre os diferentes stakeholders, incluindo actores tanto do sector público como privado, assim como as populações locais, em que todos juntam esforços para o desenvolvimento e implementação de redes em destinos turísticos (Caalders, 2003). Portanto, a construção de parcerias e a aplicação de acções e estratégias de marketing em conjunto podem reduzir o isolamento dos pequenos negócios permitindo o uso dos recursos de uma forma mais eficaz (Moutinho, 1990).

No entanto, existe pouca literatura a evidenciar que a integração das PME's que operam no sector do turismo em redes $^{3}$ possa ser usado como um factor de inovação que contribui para o desenvolvimento local (Novelli et al, 2006).

\footnotetext{
${ }^{3}$ Segundo Knoke and Kuklinski (1983, citado por Novelli et al, 2006) uma rede pode ser definida como "um tipo específico de relação que liga um conjunto de pessoas, objectos ou eventos". Porter (1998) afirma que a competitividade num mundo cada vez mais global deve focar-se nos recursos existentes a nível local, conhecimentos e relações que os competidores não consigam imitar. Mesmo nos países mais desenvolvidos esses "clusters" locais, geralmente identificáveis a nível regional, assumem um papel muito importante, sendo bem articulados a nível interno e ao mesmo tempo apoiados por uma série de factores externos que ajudam a obter
} 
Neste artigo apresentamos um case study, em que usando como base o Modelo de Albermathy e Clark verificamos os incrementos de inovação que se verificaram com a implementação de um modelo de gestão em rede em cinco regiões com destinos rurais na Europa, O objecto de estudo é o projecto Rede Europeia de Turismo de Aldeia (Genuineland) que já recebeu um prémio de inovação, Ulysses Prize, da Organização Mundial de Turismo, órgão das Nações Unidas.

\section{Inovação}

O papel da inovação nos diferentes processos económicos e na melhoria da competitividade de um país é irrefutável. Segundo a OECD (2005) a inovação é considerada como um factor que incrementa a capacidade de uma empresa para absorver e aproveitar muitos conhecimentos de todo o tipo e consequentemente manter ou incrementar a sua posição competitiva.

Existem muitas definições e suportes teóricos ligados ao termo inovação, cuja origem provém do latim innovatio (acção ou efeito de inovar) e inovar, do latim innovare, que significa mudar ou alterar as coisas introduzindo novidades (Ellul, 2008). Joseph Schumpeter é considerado o primeiro economista a dar importância à inovação (Rogers, 1998), distinguindo invenções de inovações (Hjalager, 2002). Schumpeter (1883-1950) definiu cinco tipos de inovação:

- Introdução de um novo produto ou a mudança qualitativa de um produto já existente;

- Inovação de um processo numa indústria;

- Abertura de um novo mercado;

- Desenvolvimento de novas fontes de fornecimento de matérias-primas ou outros inputs;

- Mudanças numa organização industrial.

A inovação difere da criatividade (Decelle, 2004). A criatividade está relacionada com a produção de novas ideias, novas abordagens e invenções, enquanto que a inovação corresponde à aplicação de ideias novas e criativas e à aplicação de invenções. As pessoas e as organizações podem ser criativas sem ser inovadoras.

uma posição competitiva nos mercados globais. Num período em que o conhecimento assume um papel central no processo produtivo e o ritmo de inovação tende a intensificar-se, o valor global surge a nível regional e por vezes a nível local (Nordin e Svenson, 2005). Segundo a OECD (1993) trabalhar em parceria desenvolveu-se ao mesmo tempo que surgiu um interesse crescente a nível teórico pela interacção entre stakeholders no turismo rural, as parcerias locais são cada vez mais reconhecidas como uma medida apropriada, particularmente como resposta na sua capacidade de conseguir juntar uma gama de grupos de interesse no desenvolvimento coerente de planejamento. 
Existem várias metodologias aceites internacionalmente que proporcionam marcos conceptuais para medir a actividade económica e social associada à inovação. Entre esses documentos podese citar o Manual de Frascatti (OECD, 1995), o Manual de Canberra (OECD, 1995a) e o Manual de Olso (2005). Estes manuais reflectem o amplo debate sobre a conceptualização da inovação e da sua relação com o desenvolvimento tecnológico e a actividade de investigação que se tem desenvolvido nos últimos anos (Sorzabal e Galzacorta, 2008).

\section{Inovação em Turismo}

O estudo da inovação no sector dos serviços como o turismo é complexo uma vez que a teoria e as metodologias ligadas à inovação foram principalmente desenvolvidas para a inovação tecnológica no sector industrial, não tendo em conta as particularidades do sector dos serviços (Aguiló, 2008). Existem argumentos a favor e contra o facto de se afirmar que a inovação encontrada nos serviços é diferente da encontrada na indústria (Boden e Miles, 2000). As inovações encontradas na área dos serviços incluindo o turismo centram-se mais num âmbito social ou organizacional do que na indústria onde é dado um maior foco à tecnologia (Sundbo, 1998, Gallouj, 2002). Segundo Sirilli e Evangelista (1998, citados por Jacob e Aguiló, 2008) as principais características dos serviços que têm implicações para a inovação são:

- a relação estreita entre produção e consumo;

- a intangibilidade e conteúdo intensivo em informação dos serviços e dos processos;

- o papel fundamental dos recursos humanos como factor básico de competitividade;

Geralmente as inovações em serviços são mais incrementais que radicais porque estão mais ligadas a incrementos dos processos e procedimentos que não requerem muito I\&D prévio. No entanto, essas inovações podem comportar algumas mudanças complexas que se podem centrar no apoio de stakeholders externos ou até na colaboração através de redes. Os benefícios do recurso à organização em rede no sector do turismo tem vindo a ser debatido assim como o efeito a nível da inovação (Nordin, 2003; Sundbo, 1998).

Hjalager (2002) analisou o uso do termo inovação a nível da investigação em turismo e verificou que as directrizes da inovação devem enfatizar a sustentabilidade. Esta autora adoptou os conceitos desenvolvidos por Abernathy e Clark (ver Figura 1) cujo modelo mede os níveis de inovação usando as competências essenciais como medida de análise. O modelo ilustra quatro tipos de inovação: regular, em nichos, revolucionária e arquitectural. O eixo 
vertical indica os conhecimentos e as competências usadas para o produção de bens ou serviços e o eixo horizontal indica onde as inovações específicas tornam as ligações obsoletas ou onde levam a um fortalecimento das existentes.

As inovações menos radicais são as regulares mas o seu impacto por um período longo pode, no entanto, tornar-se considerável. As inovações em nichos geralmente desafiam as estruturas colaborativas, mas não as competências básicas em termos de conhecimento. As inovações revolucionárias, por seu lado, mantêm as estruturas externas na mesma, enquanto se operam efeitos radicais nas competências. As inovações arquitecturais geralmente mudam todas as estruturas assim como estabelecem novas regras que remodelam o conceito de turismo. Este modelo fornece uma estrutura que ajuda a entender a natureza de uma inovação particular em turismo. No entanto, tem sido criticado por ser demasiado descritivo e estático.

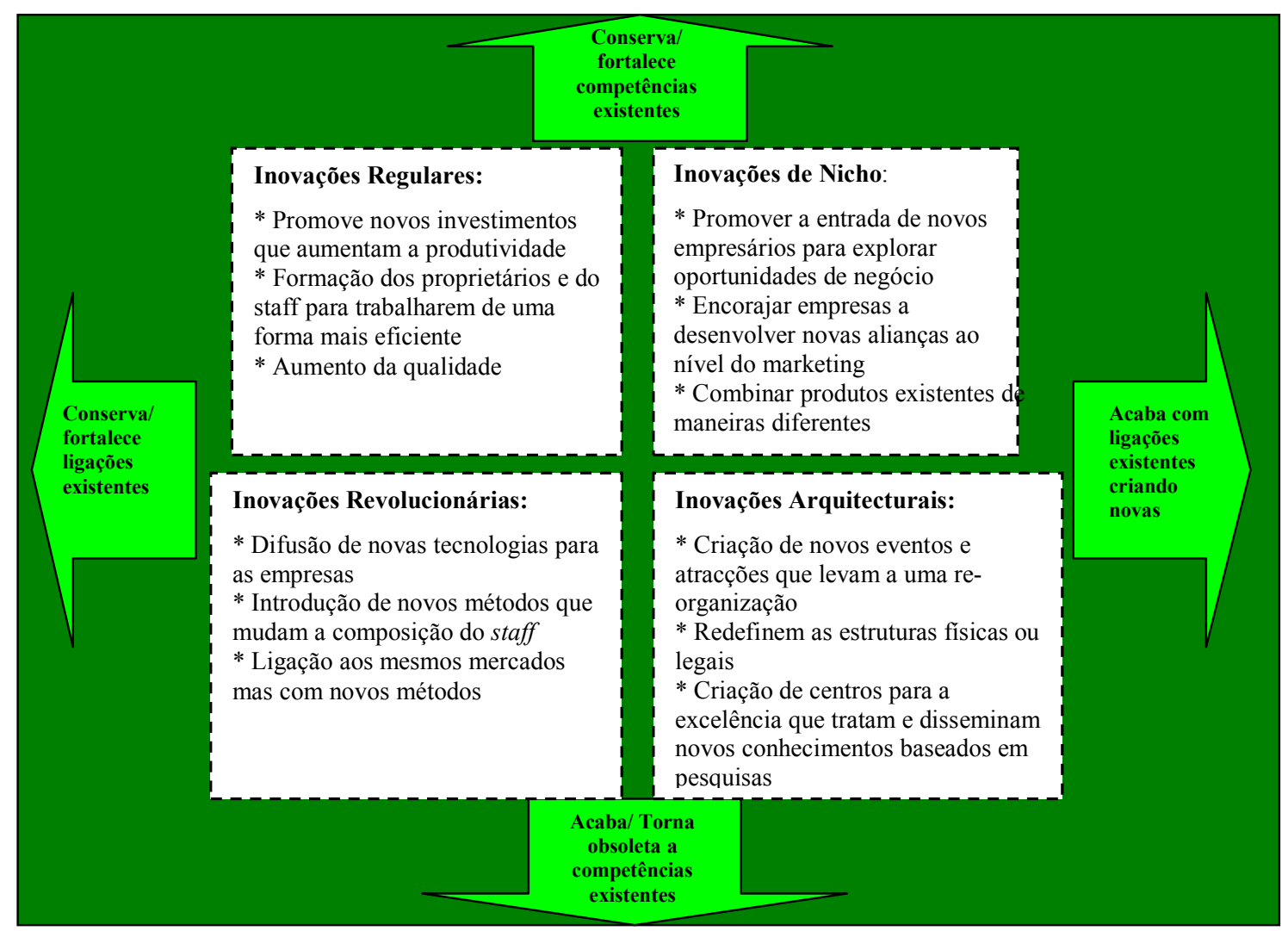

Figura 1: Modelo Abermathy and Clark

Fonte: HJALAGER, A. 2002. Repairing innovation defectiveness in tourism, Tourism Management, 23, pp 465-474 


\section{Estudo de Caso: Rede Europeia de Turismo de Aldeia}

Segundo Yin (1984) um case study é um método de pesquisa que investiga um fenómeno contemporâneo num contexto de vida real, em que as fronteiras entre a vida real e o fenómeno não são claramente evidentes e em que múltiplas fontes de provas são utilizadas. Para o estudo apresentado escolheu-se o projecto da Rede Europeia de Turismo de Aldeia e a informação foi recolhida através de entrevistas aplicadas via telefone aos diversos stakeholders envolvidos no projecto. Esta análise tem o intuito de verificar quais os incrementos de inovação que se verificaram com a implementação de um modelo de gestão em rede em cinco regiões com destinos rurais na Europa.

A ideia de criar a Rede Europeia de Turismo de Aldeia surgiu durante a execução do projecto "Learning Sustainability”, e foi desenvolvida no âmbito da iniciativa europeia Recite II. O desenvolvimento sustentável do território rural marginal Europeu tem de ser promovido para se conseguir atingir um desenvolvimento global de toda a Europa. Neste sentido, e considerando a importância que o sector do turismo pode ter para o desenvolvimento económico dessas comunidades rurais e também, e essencialmente, para o desenvolvimento social Europeu, o projecto da Rede Europeia de Turismo de Aldeia foi concebido e financiado pelo programa Interreg III C.

Para atingir o principal objectivo do projecto, o desenvolvimento sustentável das áreas rurais através do turismo, dinamizou-se uma parceria com cinco regiões localizadas en regiões marginais Europeias, nomeadamente, Alentejo em Portugal, Arad na Roménia, Lapónia na Finlândia, Lomza na Polónia e Trentino em Itália. A Rede Europeia de Turismo de Aldeia, coordenada pela Região de Turismo de Évora, foi desenvolvida através de uma parceria composta pela Universidade de Évora e a Direcção Regional de Agricultura do Alentejo em Portugal, o Centro de Ecologia Alpina e o Consórcio BIM Benta em Itália, o Centro de Educação Contínua da Universidade da Lapónia na Finlândia, a Direcção de Acção Social do Município de Arad na Roménia e o Município de Zbojna na Polónia.

No projecto "Learning Sustainability" algumas áreas de trabalho funcionaram juntas, nomeadamente, turismo, gestão de áreas naturais, qualidade, cooperativismo e relacionamento entre cidades e o ambiente rural. Este projecto foi direccionado para a pesquisa de desenvolvimento sustentável nas áreas rurais marginais da Europa, como é o caso do Alentejo em Portugal, Trentino nos Alpes Italianos e na parte da Finlândia designada por Lapónia. Na estrutura do projecto, e dado a natureza rural das três regiões, os parceiros envolvidos, e, mais especificamente os ligados à área do turismo, juntaram os seus esforços através do 
desenvolvimento de actividades nas aldeias, baseados em certas condições que serviram de base para os objectivos gerais do projecto actual mencionados anteriormente.

Entre 1999 e Setembro de 2002, a data para a conclusão do projecto "Learning Sustainability", as actividades desenvolvidas relacionadas com o turismo, na procura de formas alternativas ao turismo de massas, permitiram o estabelecer das bases para o lançamento da Rede Europeia de Turismo de Aldeia. A selecção dos critérios, intervenção e as estratégias de marketing levaram a parceria a criar um produto turístico baseado em aldeias, nas suas tradições, no seu património assim como o fundamento de que tinha como base os principios de sustentabilidade e a integração de todas as actividades.

Apesar da Rede Europeia não estar incluída como um dos primeiros produtos do projecto "Learning Sustainability", o ideal que ela representava levou a que os parceiros juntassem esforços com o objectivo de criar uma Rede de turismo a um nível Europeu como uma base de trabalho e de comunicação com outras áreas de trabalho do projecto. Para além disso, o estabelecimento de uma Rede Europeia de Turismo de Aldeia sob a coordenação do grupo de trabalho do Alentejo, levou à construção de algo mais tangível tendo como base uma sólida parceria, desenvolvendo um dos produtos do projecto com mais futuro e capacidade de continuidade.

Como consequência desta ideia e das relações estabelecidas sob a alçada do projecto "Learning Sustainability" combinado com outros projectos envolvendo Arad (Roménia) e Zbójna (Polónia) duas regiões com objectivos similares (projecto Intregradev), foi estabelecida uma parceria para concretizar a ideia de criar uma Rede de Turismo de Aldeia. Esta parceria apresentou uma candidatura, em Janeiro de 2003, à iniciativa Interreg III C Sul, que foi aprovada em Junho do mesmo ano. Como resultado, nasceu um novo período do projecto que durou até ao final de 2006 e que ficou com potencial para continuar no futuro. As regiões incluídas no projecto Interreg III C, Alentejo (Portugal), Lapónia (Finlândia), Trentino (Italia), Arad (Roménia) e Lomza (Polónia), estavam a confrontar-se com dificuldades características das zonas rurais periféricas da Europa. 


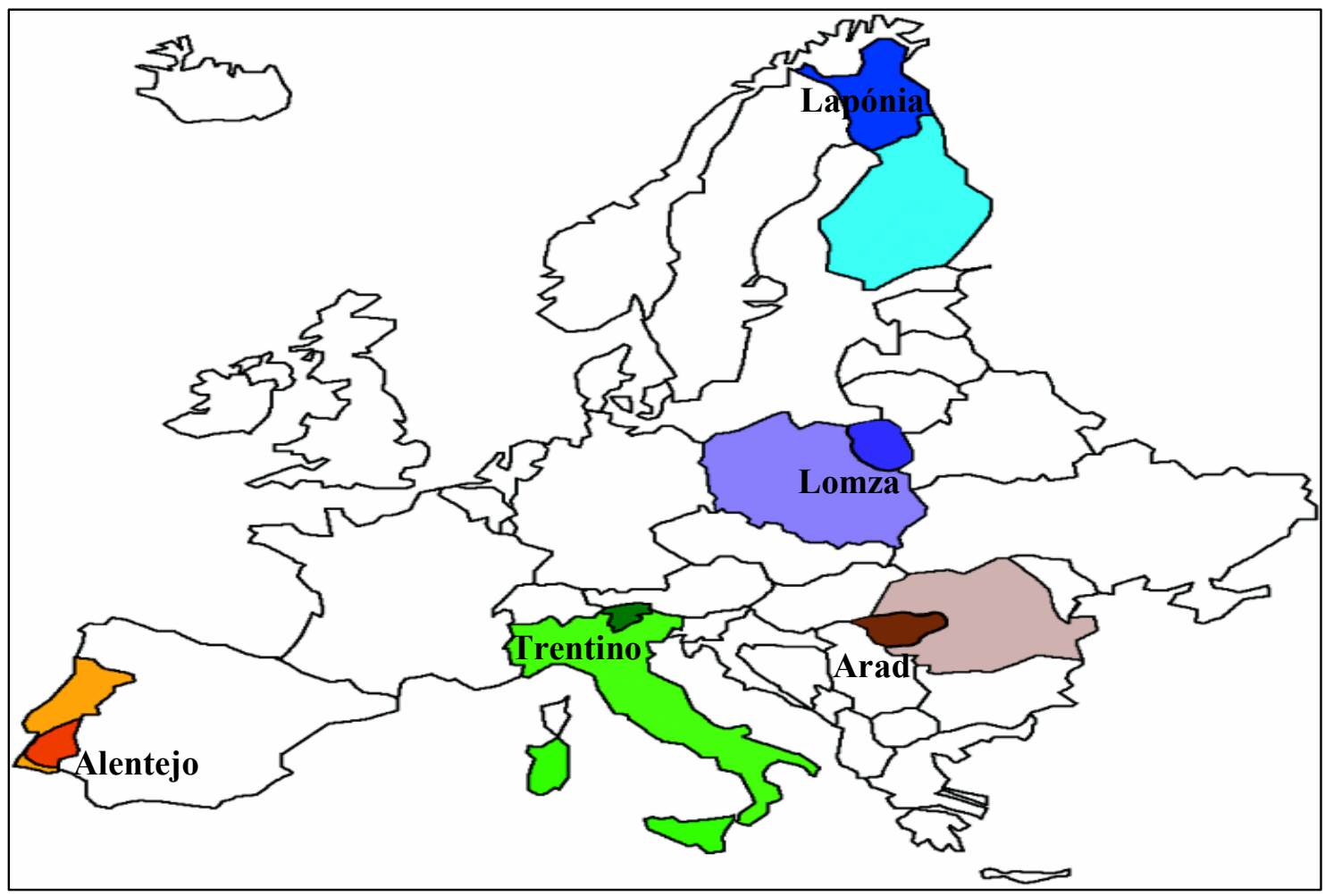

Figura 2: Regiões e aldeias pertencentes à Rede Europeia de Turismo de Aldeia Fonte: RTE, 2006

Apesar destas regiões serem claramente diferentes em termos geográficos, culturais e sociais, têm em comum o facto de muitas delas serem localizadas em áreas rurais marginais da Europa que enfrentam grandes limitações em termos de desenvolvimento, causadas pelo aumento do isolamento, degradação das actividades económicas e o consequente aumento do desemprego. Para além disso estas regiões estão a sofrer o fenómeno do envelhecimento da população e da desertificação humana, assim como outros factores que influenciam negativamente o seu processo de desenvolvimento. Muitas das pessoas que nasceram nessas regiões não têm possibilidade de ficar e consequentemente emigram para os centros urbanos.

Desde então tem sido difícil atrair novos residentes resultando num grande decréscimo da população levando mesmo ao desaparecimento de algumas comunidades. Para contrariar esta tendência, é necessário promover iniciativas que contribuam para um desenvolvimento sustentável dessas regiões. Apesar dos constrangimentos, essas regiões possuem um grande potencial sendo áreas com uma forte preservação ambiental e que possuem uma vasta riqueza cultural e etnográfica que pode ser usada para estas iniciativas. 
Foi neste contexto que a Rede Europeia de Turismo de Aldeia foi desenvolvida, usando o turismo como um catalizador da sustentabilidade, através da promoção dos recursos endógenos e do desenvolvimento turístico nas aldeias integradas e na criação de estruturas sustentáveis para a cooperação europeia neste domínio. A parceria criada baseia-se num sistema sólido de rede não só a nível internacional mas também a nível regional, combinado com uma perspectiva bottom-up no desenvolvimento de actividades em que as comunidades são envolvidas.

Uma vez que o desenvolvimento endógeno não pode ser atingido sem um conhecimento profundo das realidades locais, foi estabelecido que seria importante a elaboração de planos de aldeia (Planos de desenvolvimento local) para o estabelecimento da rede e de todas as actividades que iriam ser desenvolvidas em cada aldeia integrada na rede. Adicionalmente, foi considerado relevante o desenvolvimento de um plano para a actividade turística da rede com o intuito de consolidar o novo produto turístico assim como de desenvolver uma estratégia de informação e promoção. Outro aspecto considerado importante centrou-se na realização de acções de formação e qualificação profissional, com o intuito de dar às comunidades locais e outros actores capacidades para promover o seu capital social.

Neste sentido, este projecto junta um plano de desenvolvimento local (plano de aldeia), complementado por análises de formação, com o intuito de fornecer as bases para um plano de formação e para um plano de actividades turísticas. As actividades do projecto procuram o desenvolvimento dos conceitos de "Turismo de Aldeia" e de "Turismo do Imaginário" 4 , como partes das actividades turísticas desenvolvidas nas regiões integradas.

Como uma base para o desenvolvimento de actividades inseridas no projecto, foi inicialmente concebido um documento guia que aprofundou o conceito de turismo de aldeia, estabelecendo os critérios para a selecção das aldeias, os Planos de Aldeia para cada uma delas e um plano estratégico para o desenvolvimento da rede, que definiu a estrutura e o modelo para gestão e financiamento, assim como estratégias de marketing. Ao mesmo tempo que se desenvolveu estes relatórios, efectuou-se uma análise dos perfis dos utilizadores finais, não só para as aldeias e regiões envolvidas mas fazendo-se uma comparação com outros tipos de

\footnotetext{
4 "Turismo do Imaginário" refere-se à descoberta do território através de narrativas moldadas pela cultura material e imaterial das comunidades. É uma marca registada pela Região de Turismo de Évora no contexto da Rede Europeia de Turismo de Aldeia As cinco regiões envolvidas exploram diferentes temas, como o Megalitismo no Alentejo, O Shamanismo na Lapónia, a bruxaria na Itália, a arvore da vida em Lomza e as lendas da montanha em Arad. Dentro de cada tema são desenvolvidas diversas actividades como redes de trilhos pedestres, seminários, eventos, teatro de rua, workshops, entre outros.
} 
experiências oferecidas na Europa. Estes documentos servem de base não só para estabelecer a rede, mas também para fornecer um caminho para as actividades de informação e promoção, assim como para as actividades relacionadas com a formação.

Foi também criada uma marca que promove de uma forma distintiva todo o projecto, designada "Genuineland”. Ao mesmo tempo, foram criados produtos turísticos estimulantes sob a temática "Turismo do imaginário", que levou ao envolvimento das populações locais, de uma forma voluntária, em actividades como recrear eventos históricos, viagens organizadas, organização da oferta turística e actividades conjuntas ou intercâmbios com outras aldeias.

O esforço contínuo para estimular e fortalecer a rede foi amplamente desenvolvido entre 2003 e 2007, baseado em encontros que envolviam os representantes de todos os parceiros.

Os encontros desenrolaram-se em cada uma das regiões participantes do projecto, dinamizando um leque de objectivos: coordenação do projecto global; aprendizagem sobre as experiências de intercâmbio; seminários temáticos centrados em questões específicas ligadas aos processos de desenvolvimento local; acções de benchmarking entre regiões parceiras. Para além disso, estes encontros foram um meio de discutir e avaliar os documentos de orientação e de preparar a organização dos encontros regionais fundamentais ao desenvolvimento de uma rede e das necessárias competências para a criação de laços de cooperação.

Estas acções permitiram, através de trocas intensivas de experiências, a consolidação das bases de cooperação, permitindo a disseminação dos resultados das experiências mais importantes em cada comunidade ao nível do desenvolvimento sustentável. Isto também permitiu o desenvolvimento de actividades de formação profissional e de promoção, com o objectivo de aumentar a qualidade da gestão dos produtos locais e particularmente da oferta turística da aldeia.

Em 2007 a rede avançou para a formalização através da criação de uma associação sem fins lucrativos e gerou um grande entusiasmo junto de outras regiões interessadas em desenvolver este projecto no seu território. Às cinco regiões iniciais juntaram-se mais cinco regiões oriundas da Grécia, Eslovénia, Bélgica e Itália (Toscânia e Piemonte). Ainda em 2007 e em reconhecimento da inovação proporcionada por este projecto a Organização Mundial de Turismo, órgão das Nações Unidas, premiou o projecto com o Prémio Ulysses 2007.

O projecto de criação de uma Rede Europeia de Turismo de Aldeia introduziu um grande número de inovações nos territórios envolvidos que são apresentadas sumariamente de acordo 
com o modelo de Abernathy e Clark. No entanto o processo é contínuo e outras inovações estão em curso para os territórios e comunidades envolvidas.

\begin{tabular}{|c|c|}
\hline $\begin{array}{c}\text { Nível de Inovação } \\
\text { Segundo Abernathy e } \\
\text { Clark }\end{array}$ & Inovação \\
\hline Regular & $\begin{array}{l}\text { Criação de standards de qualidade com base na marca } \\
\text { Genuineland. } \\
\text { Criação de um plano de formação-acção personalizado para } \\
\text { todos os membros da Rede. } \\
\text { Envolvimento das regiões e respectivas comunidades em } \\
\text { todas as fases do projecto. }\end{array}$ \\
\hline Nicho & $\begin{array}{l}\text { Crescimento de } 40 \% \text { em novos investimentos ao nível do } \\
\text { alojamento, animação, artesanato e restauração. } \\
\text { Reconhecimento da necessidade de cooperar. } \\
\text { Criação de uma rede de clusters dentro da Rede com } \\
\text { objectivos específicos de marketing. } \\
\text { Criação de ofertas temáticas com base nos recursos e na } \\
\text { criação de novos elementos e combinações. } \\
\text { Criação de uma marca única: Genuineland. }\end{array}$ \\
\hline Revolucionária & $\begin{array}{l}\text { Maximização dos mercados existentes e penetração em novos } \\
\text { mercados com recurso ao Turismo do Imaginário, criação da } \\
\text { Rede. } \\
\text { Modelo de promoção baseado em sinergias nacionais e } \\
\text { internacionais. }\end{array}$ \\
\hline Arquitectural & $\begin{array}{l}\text { Redefinição do modelo de comercialização dos pacotes. } \\
\text { Modelo específico para a criação de eventos. } \\
\text { Criação de um modelo organizacional específico cuja base } \\
\text { assenta na cooperação. }\end{array}$ \\
\hline
\end{tabular}

Figura 3: Inovações introduzidas nas aldeias com a implementação da Rede Europeia de Turismo de Aldeia

Fonte: autor

\section{Conclusão}

A Rede Europeia de Turismo de Aldeia permitiu a unificação de esforços de micro e pequenas empresas em torno de uma ideia, de um projecto. O reconhecimento dos benefícios resultantes da cooperação ao nível local, regional e internacional por parte dos actores envolvidos criou condições para o desenvolvimento de estratégias que de outra forma seriam impossíveis de implementar. Com esta mudança de atitude é possível criar e desenvolver programas de melhoria da qualidade do destino, programas específicos de formação, novas estratégias de marketing, implementar eventos baseados nos recursos da comunidade e implementar novos modelos organizacionais, sem nunca esquecer a diversidade cultural e espacial. É com base 
neste reconhecimento que foi possível criar uma Rede de cooperação cujo objectivo primordial é contribuir para a melhoria dos destinos envolvidos e para uma promoção mais eficaz baseada por um lado na unificação de esforços e por outro na diversidade e diferença que torna este produto atractivo.

A parceria e as comunidades envolvidas reconheceram a necessidade de inovar, de introduzir melhorias nos processos, de criar e adaptar a oferta para um mercado cada vez mais competitivo e com um crescente número de nichos de mercado que é necessário dar resposta. É com esta filosofia que o projecto continua a crescer e a envolver novas regiões.

\section{Referências}

BODEN, M. e MILES, I. (eds.).2000. Services and the Knowledge-Based Economy (London: Continuum).

BREDA, Z; COSTA, R. e COSTA, C. 2006. Do Clusters and Networks Make Small Places Beautiful? The Case of Caramulo (Portugal)" in LAZZERETTI, L. e PETRILLO, C. ed. Tourism Local Systems and Networking, Advances in Tourism Management Series. Oxford: Elsevier, pp. 67-82.

CAALDERS, J. 2003. Tourism in Freesland: a network approach in HALL, D. e RICHARDS ed. Tourism and Sustainable Community Development, , UK: Routledge pp 185-205.

DECELLE, X. 2004. A conceptual and dynamic approach innovation in tourism. OECD. Disponível em: http://www.oecd.org/dataoecd/55/28/34267921.pdf.

DINIS, F. 1999. Rural Tourism Impact in Local Economies - proportional income and employment multipliers (The case of Douro Region). Summer Institute 1999, Tourism Sustainability and Territorial Organization, Faro.

ELLUL, D. 2008. Inovación en Turismo, pólo gastronómico Tomás Jofré - Buenos Aires, Argentina, ROTUR. Revista de Ócio e Turismo, Vol. 1, pp.

FRA, M. 2004. A iniciativa comunitária LEADER no marco teórico da política agrária. Revista Galega de Economia. Vol 13 (1-2), 175-198.

GALLOUJ, F. 2002 Innovation in the Service Economy. (Cheltenham: Elgar).

HJALAGER, A. 2002. Repairing innovation defectiveness in tourism, Tourism Management, 23, pp 465-474.

JACOB, M. e AGUILÓ, E. 2008. La innovación en el sector turístico: El caso de Baleares, ROTUR, Revista de Ócio e Turismo, Vol 1, pp 51-64.

MOUTINHO, L. 1990. Strategies for Tourism Destination Development: an Investigation of the Role of Small Businesses in G. ASHWORTH and B. GOODALL ed. Marketing Tourism Places. Routledge, London, New York, 104-122.

NORDIN, S. and SVENSON, B. 2005. The significance of Governance in Innovative Tourism Destinations, Östersund: European Tourism Research Institute.

NOVELLI, M., SCHMITZ, B. and SPENCER, T. 2006. Networks, clusters and innovation in tourism: A UK experience. Tourism Management, 27, 1141-1152. 
OECD .1995. Manual de Frascatti. Ed. OECD. Disponível em: $<$ http://www.mct.gov.br/index.php/content/view/4029.htm>.

OECD .1995a. Manual de Canberra. Ed. OECD. Disponível em: $<$ http://www.oecd.org/dataoecd/34/0/2096025.pdf>.

OECD .2005. Manual de Olso. Ed. OECD. Disponível em: $<$ http://www.finep.gov.br/imprensa/sala_imprensa/manual_de_oslo.pdf $>$.

OECD .2005. OECD and entrepreneurship outlook 2005, OECD.

ROGERS, M. 1998. The definition and measurement of innovation, Melbourne Institute Working Paper. Disponível em: <http://www.melbourneinstitute.com/wp/wp1998n10.pdf>.

SHARPLEY, R. 2005. Managing the countryside for tourism: a governance perspective in L. PENDER R. SHARPLEY ed. The Management of Tourism. Sage Publications, 175-186.

SORZABAL; A e GALZACORTA, M. 2008. Innovación en la actividad turística vascã: hacia um modelo competitivo en la C.A. de Euskadi, ROTUR, Revista de Ócio e Turismo, Vol. 1. pp 65-86

SUNDBO, J. 1998. The Organization of Innovation in Services (Copenhagen: Roskilde University Press).

WILSON, S.; FESENMIER, D.; FESENMEIER, J. and VAN ES, J. 2001. Factor for success in Rural Tourism Development. Journal of Travel Research, Vol. 40 (2), 132-138.

YIN, R.K. 1984. Case Study Research: Design and Methods. Beverly Hills, Calif: Sage Publications.

\section{Recebido em: 02/06/2008}

Aprovado em: 12/09/2008 